\title{
Impactos da Pandemia de Covid-19 sobre Profissionais de Gestão de Pessoas
}

\author{
Impacts of the Covid-19 Pandemic on People Management Professionals \\ Impactos de la Pandemia Covid-19 en los Profesionales de la Gestión de Personas
}

\author{
Isabella Fernanda Rodrigues Felipe \\ Vinícius Ramalho Medeiros \\ Mário Lázaro Camargo ${ }^{1}$ \\ Edward Goulart Júnior \\ Universidade Estadual Paulista Júlio de Mesquita Filho (UNESP)
}

\begin{abstract}
Resumo
Este estudo objetivou compreender como a pandemia de covid-19 impactou as relações de trabalho e os profissionais da área de gestão de pessoas, no tocante às medidas de contenção ao contágio. A pesquisa cumpriu um protocolo de triangulação metodológica, utilizando instrumentos de coleta de dados e técnicas de análise próprios da pesquisa quantiqualitativa: análise de conteúdo e estatística descritiva. Participaram da pesquisa 60 profissionais de diferentes cidades brasileiras, sendo que $60 \%$ deles são psicólogos organizacionais e do trabalho. No tocante aos impactos psicológicos e físicos relatados, destacam-se: episódios de ansiedade; irritabilidade; mal-estar físico; pressão para manutenção ou aumento do desempenho; medo de perder o emprego; dificuldade em manter o equilíbrio na relação trabalho-família. Tal quadro coloca em alerta as organizações, que deverão ser impactadas pela situação de saúde física e mental dos seus profissionais, com prováveis quedas de desempenho e aumento nos índices de absenteísmos e rotatividade.
\end{abstract}

Palavras-chave: covid-19, gestão de pessoas, saúde do trabalhador

\begin{abstract}
This study aimed to understand how the covid-19 pandemic impacted work relationships and the people management professionals in terms of contention measures for contagion. The research complied with a methodological triangulation protocol using data collection instruments and analysis techniques guided to quantitative and qualitative research: content analysis and descriptive statistics. Sixty professionals from different Brazilian cities participated in the research, $60 \%$ of whom are work and organizational psychologists. Regarding the reported psychological and physical impacts, the following stand out: episodes of anxiety; irritability; physical malaise; pressure to maintain or increase performance; fear of dismissal; difficulty maintaining balance in the work-family relationship. This situation puts organizations on alert, which should be impacted by the physical and mental health of their professionals, with probable falls in their performance and an increase in absenteeism and turnover rates.
\end{abstract}

Keywords: covid-19, people management, workers' health

\section{Resumen}

Este estudio tuvo el objetivo de comprender cómo la pandemia de covid-19 impactó las relaciones laborales de los gerentes de personas debido a las medidas para contener su contagio. La investigación siguió un protocolo de triangulación metodológica utilizando instrumentos de recolección de datos y técnicas de análisis propias de la investigación cuantitativa y cualitativa: análisis de contenido y estadística descriptiva. Participaron de la investigación 60 profesionales de diferentes ciudades brasileñas, de los cuales el 60\% son psicólogos organizacionales del trabajo. Acerca de los impactos psicológicos y físicos reportados, se destacan los siguientes: episodios de ansiedad; irritabilidad; malestar físico; presión para mantener o aumentar el rendimiento; miedo de desempleo; dificultad para mantener el equilibrio en la relación trabajo-familia. Esta situación pone las organizaciones en alerta, que deberán verse impactadas por la situación de salud física y mental de sus profesionales, con probables caídas en su desempeño y un aumento de las tasas de absentismo y rotación Palabras clave: covid-19, gestión de personas, salud del trabajador

\footnotetext{
${ }^{1}$ Endereço de contato: Faculdade de Ciências, Campus de Bauru, Departamento de Psicologia - Av. Eng. Luiz Edmundo Carrijo Coube, 14-01, Vargem Limpa, Bauru, SP. CEP 17033-360. Telefone: (14) 3276-1994 / 99142-3543. E-mail: mario.camargo@unesp.br
} 


\section{Introdução}

De acordo com a Organização Mundial da Saúde (OMS, 2020a) e o Ministério da Saúde (Brasil, 2020a), a Coronavirus Disease - 2019 (covid-19) é uma doença causada pelo novo coronavírus SARS-CoV-2, cujos principais sintomas definem-na como uma infecção respiratória, podendo sua sintomatologia variar de pessoa para pessoa, desde situações de acometimentos assintomáticos até casos mais graves com óbito. Em 11 de março de 2020, a OMS caracterizou a covid-19 como sendo uma pandemia e, em maio de 2020, já estava presente em 215 países. No Brasil, de acordo com dados do Ministério da Saúde (Brasil, 2020b) atualizados no dia 17 de setembro de 2020, a doença já ultrapassava os 4.419 .083 de casos confirmados e 134.106 óbitos.

Considerando-se o fato de que a covid-19 espalha-se facilmente entre os seres humanos, Manandhar, Nakami e Baniya (2020) argumentam que quase todos os países do mundo estão lançando mão de estratégias para contenção do avanço da pandemia por meio de medidas sanitárias e de higiene, utilização de máscaras protetoras e evitação do contato social e físico. Estas práticas vêm sendo chamadas de "isolamento e distanciamento social" pelas instituições de saúde, governos e imprensa (Organização Mundial da Saúde, 2020b; Ministério da Saúde, 2020a). Nesse cenário, diversos aspectos da vida cotidiana foram afetados, como o lazer, a educação e, também, as relações de trabalho. Pinheiro (2020) comenta que o ineditismo da situação tem sido um empecilho no contexto organizacional, dificultando os negócios e colocando em risco os empregos.

Diferentes ações foram sugeridas pela OMS (2020a) e pela Secretaria de Inspeção do Trabalho (2020) para o ambiente de trabalho, são elas: manter as mãos, as superfícies, o ar e o ambiente como um todo bem higienizados; manter distância segura entre os trabalhadores (de 1,5 metro a 2 metros), dividindo-os em turnos com menos pessoas, caso necessário; diminuir a frequência e a duração do contato com o público externo, agendando horários para evitar aglomerações, com adequação dos processos produtivos, entre outras, todas com significativo impacto nos contextos e nas relações de trabalho. Mudanças repentinas e acentuadas causam reações emocionais nas pessoas e, segundo Cullen, Gulati e Kelly (2020), a necessidade de cuidados psicológicos e psiquiátricos devem ser considerados.

A pandemia de covid-19 está causando mudanças e questionamentos sobre o mundo do trabalho, com consequências para diferentes segmentos. As pessoas que trabalham nas mais variadas atividades, mas especialmente as que atuam dentro do chamado mercado informal (e.g., vendedores ambulantes, prestadores de serviços em manutenção, trabalhadores domésticos etc.), veem-se impedidas de trabalhar, em decorrência do distanciamento social. Outro segmento fortemente impactado são os profissionais de saúde, principalmente aqueles que trabalham na linha de frente do combate à pandemia; esses estão atuando sob forte sobrecarga emocional e acentuadas demandas de trabalho (Faro et al., 2020). De maneira geral, a quase totalidade das organizações de trabalho tiveram de rever seus procedimentos de atuação, bem como suas políticas e práticas de gestão organizacional.

As exigências no combate ao contágio da covid-19 nos contextos de trabalho colocaram a área de Gestão de Pessoas (GP) em evidência, considerando que a área realiza diferentes funções, que vão desde ações de seleção de pessoas, até políticas de desenvolvimento, remuneração, legalização das relações trabalhistas, programas de qualidade de vida no 
trabalho e saúde do trabalhador (Castro, Oliveira, Morais, \& Gai, 2020). As mudanças decorrentes da situação vivenciada impactaram sobremaneira os trabalhadores que tiveram de se adaptar a novas rotinas e procedimentos do trabalho. O protagonismo dos profissionais que atuam em GP nesta pandemia ratificou a importância da área na proposição, no planejamento e no gerenciamento das mudanças impostas, adequando e capacitando os profissionais para o exercício de diferentes atividades, que, para muitos, são inéditas e inesperadas.

A atuação em GP não exige uma formação específica, abarcando profissionais formados e graduados em diferentes ciências (e.g., Psicologia, Administração de Empresas, Serviço Social, Contabilidade, Tecnologia em Recursos Humanos etc.). A multidisciplinariedade da área, especialmente a que se vivencia na atualidade, possibilita um olhar sobre o fenômeno mais ampliado e sistêmico, favorecendo uma atuação mais qualificada diante das demandas atuais nos contextos de trabalho (Zanelli, Bastos, \& Rodrigues, 2014). Entre a equipe interdisciplinar que compõe a área, destaca-se a figura do Psicólogo Organizacional e do Trabalho (POT), o qual é apresentado por Marinho e Camargo (2017) como um profissional atuante, como pesquisador e/ou promotor de intervenções, na relação trabalhador-organização-trabalho, objetivando promover saúde, desenvolvimento humano e profissional.

Este estudo teve o objetivo de compreender como a pandemia da covid-19 influencia as relações de trabalho e os trabalhadores, em especial, aqueles que atuam nas áreas de GP de organizações públicas e privadas (psicólogos, administradores de empresas, assistentes sociais, contadores, tecnólogos em recursos humanos etc.), no tocante às medidas de contenção necessárias para diminuir a propagação do novo coronavírus na organização em que atuam.

\section{Método}

Esta pesquisa cumpriu um protocolo de triangulação metodológica ao aplicar instrumentos de coleta de dados e se utilizar de técnicas de análise próprios da pesquisa quantitativa e qualitativa em ciências sociais (Azevedo, Oliveira, Gonzales, \& Abdalla, 2013), em especial, a técnica de análise de conteúdo, para as questões dissertativas (Bardin, 2011), e a estatística descritiva, para os dados quantitativos (Vendramini, 2016).

\section{Procedimentos e Instrumentos}

Considerando a complexidade do objeto de estudo eleito e para abordá-lo de forma efetiva, optamos por preparar e aplicar instrumentos que coletam e quantificam frequências de respostas, tanto quanto permitem a análise dos significados atribuídos pelos participantes ao fenômeno estudado. Aplicamos, primeiramente, um questionário sociodemográfico, com o objetivo de coletar dados sobre os participantes da pesquisa e traçar um perfil deles em termos de: idade, gênero, formação acadêmica e qualificação profissional, tipo de vínculo profissional (se autônomo, da iniciativa privada ou servidor público), localização de residência e trabalho no país (estado e cidade), denominação do cargo em exercício na organização e número de pessoas impactadas por seus projetos e decisões etc.

Em seguida, aplicamos um questionário estruturado com perguntas dissertativas e objetivas sobre como as medidas de contenção à pandemia de covid-19 têm influenciado a dinâmica de trabalho dos participantes da pesquisa e impactado sobre suas emoções. Foram 
abordadas as formas de enfrentamento à pandemia que os participantes vêm colocando em prática na atividade profissional e na organização, havendo, portanto, questões específicas dirigidas aos participantes que atuavam, no momento da pandemia, em sistema de teletrabalho (home office) e outras questões dirigidas a profissionais que atuavam presencialmente, dado que nem todas as organizações de trabalho interromperam suas atividades presenciais.

Adotamos as seguintes estratégias para coleta de dados: a) foram convidados a participar da pesquisa os profissionais atuantes em GP. Os questionários foram formulados e apresentados aos participantes por meio do aplicativo de pesquisa Google Forms, que permite recebimento, preenchimento e envio on-line. Para além das questões referentes aos dados sociodemográficos e objetivas (em número de 14), as questões dissertativas (em número de 8) versaram sobre: quais medidas de contenção ao contágio foram adotadas por sua organização de trabalho (?); que impactos essas medidas geraram sobre sua rotina laboral, condição de saúde física e mental (?); quais os efeitos (positivos ou negativos) possíveis da situação de pandemia sobre sua condição emocional (?); considerando um cenário pós-pandêmico, que mudanças ocorridas durante a pandemia poderão permanecer em seu contexto organizacional, em sua atuação profissional e vida pessoal (?).

Para a finalização do processo de coleta e organização dos dados, três regras foram aplicadas, entre as quais se impôs a que primeiramente ocorreu, a saber: 1. atingir o número de 60 participantes (amostra por conveniência entre os dias 18 de maio e 18 de junho de 2020) com Termos de Consentimento Livre e Esclarecido (TCLE) assinados e questionários devidamente preenchidos e enviados aos pesquisadores; 2 . vencer o período de 30 dias de prazo, contado a partir do envio do convite para a participação na pesquisa; 3. mediante alcance do ponto de saturação nas respostas abertas e submetidas à análise de conteúdo (Bardin, 2011; Fontanella, Ricas, \& Turato, 2008), ou seja, interrompemos o recebimento dos questionários a partir da percepção de que os dados encontrados se repetiam, apesar das variações da fonte, mantendo-se estável como conteúdo no processo de análise de conteúdo empreendido.

\section{Procedimentos Éticos}

Em se tratando dos cuidados éticos relativos à pesquisa envolvendo seres humanos, importa informar que a presente pesquisa teve seu projeto submetido e aprovado por um Comitê de Ética em Pesquisa (CAAE n. 31675020.9.0000.5398), conforme orientações presentes nas Resoluções 466/12 e 510/16, do Conselho Nacional de Saúde, e os participantes deram seu aceite mediante assinatura do TCLE.

\section{Resultados e Discussões}

A partir de um formulário on-line disponibilizado via redes sociais, chegou-se a 60 respostas para realização da análise. Dos 60 participantes, 76,7\% eram mulheres ( $n=46)$ e 23,3\%, homens $(n=14)$, sendo que a maioria dos pesquisados estão na faixa etária de 30 a 40 anos.

Quanto à formação acadêmica, $85 \%$ possuem ensino superior completo $(n=51)$, sendo que, desses, $60 \%$ cursaram Psicologia $(n=36) ; 17 \%$, Administração de Empresas $(n=10)$; e os demais são graduados em diferentes cursos, como Direito, Engenharia de Produção, 
Ciências Contábeis etc. Aproximadamente 70\% $(n=42)$ dos pesquisados possuem curso de pós-graduação Lato ou Stricto Sensu, a maioria em GP ou Psicologia Organizacional e do Trabalho (POT). Os dados apresentados indicam a multidisciplinariedade que compõe a área de GP nas organizações.

Dos participantes do estudo, 27 pessoas $(46,7 \%)$ relataram ser profissionais vinculados à iniciativa privada; 22 respostas $(36,7 \%)$ vieram de profissionais da iniciativa pública; cinco profissionais $(8,3 \%)$ se apresentaram como autônomos; e 8,3\% são profissionais de áreas distintas $(n=5)$, como de Empresas Juniores, sócio de empresa de consultoria de GP etc. O cargo com maior número de participantes foi de analista de recursos humanos ( $\mathrm{RH}$ ), com 14 participantes (23,3\%); seguido de supervisor de RH (18,3\%, $n=11)$; POT $(8,3 \%, n=5)$; gerente de GP $(11,7 \%, n=7)$; assistente de $\mathrm{RH}(6,7 \%, n=4)$. Os restantes têm ocupações diversas dentro das organizações onde atuam.

Parte considerável dos participantes (40\%) trabalham em organizações com mais de mil trabalhadores ( $n=24) ; 18,3 \%$ trabalham em organizações com 201 a 500 trabalhadores $(n=11) ; 16,7 \%$, em organizações com 101 a 200 trabalhadores $(n=10) ; 13,3 \%$, de 0 a 20 trabalhadores $(n=8) ; 6,7 \%$ trabalham em organizações com 501 a 1.000 trabalhadores $(n=4)$; $3,3 \%$ de 21 a 50 trabalhadores $(n=6)$; apenas um participante trabalha em uma organização com 51 a 100 trabalhadores.

Os participantes do estudo atuam em diferentes segmentos, tais como, indústria, comércio, prestação de serviços, agronegócios, saúde, logística etc., perfazendo, portanto, uma amostragem bem diversificada. A maior parte dos pesquisados informou que sua organização, durante a pandemia, encontrava-se com as atividades parcialmente paralisadas $(56,7 \%$, $n=34) ; 30 \%$ continuaram trabalhando normalmente, sem sofrer influência da pandemia ( $n=18)$; e, por fim, $11,7 \%$ afirmaram que a organização estava com as atividades totalmente paralisadas $(n=7)$.

Sobre as medidas de contenção que as organizações puseram em prática no período da pandemia, $71,7 \%$ dos participantes da pesquisa relataram que a organização adotou o teletrabalho ( $n=43)$; 35\% afirmaram que os trabalhadores foram colocados em férias antecipadas $(n=21) ; 20 \%$ disseram que as organizações reduziram salários para manter os empregos $(n=12) ; 18,3 \%$ afirmaram que a organização suspendeu contratos de trabalho $(n=11)$; $18,3 \%$ afirmaram que as organizações fizeram demissões durante o período $(n=11) ; 6,7 \%$ relataram que a organização adotou sistema de férias coletivas $(n=4)$; e, por fim, houve respostas isoladas apontando outras medidas, como: afastamento apenas de trabalhadores de grupo de risco, redução da jornada de trabalho, desconto em banco de horas etc.

Quando questionados sobre o expediente de trabalho no período, grande parte dos participantes $(43,3 \%)$ estava em modalidade de teletrabalho $(n=26) ; 26,7 \%$ estavam dividindo a jornada entre teletrabalho e modo presencial $(n=16)$; enquanto isso, $21,7 \%$ continuavam indo à organização todos os dias $(n=13)$. No que tange às medidas de contenção de contágio adotadas dentro da organização, a quase totalidade dos participantes relataram a disponibilização de EPIs para os trabalhadores, além do álcool 70\%, em gel. Ainda, 68,3\% dos respondentes indicaram que foram ampliados os cuidados e as medidas de higiene $(n=41), 60 \%$ afirmaram que foi limitado o fluxo de pessoas dentro da organização $(n=36), 48,3 \%$ participantes informaram também a alteração de horários e turnos de trabalho $(n=29)$ e 28,3\% indicaram o redesenho do layout do espaço de trabalho, a fim de promover o distanciamento 
entre os trabalhadores $(n=17)$. Foi possível constatar também que outras medidas foram adotadas, como a oferta do serviço de teleacolhimento, mudanças em layout de refeitórios e reuniões on-line com maior frequência.

No tocante aos impactos psicológicos e físicos advindos da pandemia, os resultados mostraram que a maioria dos participantes $(65 \%, n=39)$ experimentou episódios de ansiedade; $53,3 \%$ se perceberam com maior irritabilidade $(n=32)$; $50 \%$ sentiram algum tipo de mal-estar físico, como dor de cabeça, dor no corpo, tontura, tremor etc. $(n=30) ; 38,3 \%$ relataram aumento na ingestão de alimentos nesse período $(n=23) ; 35 \%$ foram acometidos por episódios de insônia e percepção de ganho de peso $(n=21)$; 35\% sentiram-se pressionados para manutenção ou aumento do desempenho no trabalho; $31,7 \%$ relataram o medo de perder o emprego ( $n=19$ ); 30\% tiveram a dificuldade aumentada em manter o equilíbrio na relação trabalho-família ( $n=18$ ); $15 \%$ relataram conflitos nos relacionamentos afetivos $(n=9) ; 13,3 \%$ relataram conflitos nos relacionamentos familiares $(n=8) ; 13,3 \%$ relataram aumento na ingestão de medicamentos; $8,3 \%$ vivenciaram aumento no consumo de bebidas alcoólicas ( $n=5) ; 6,7 \%$ tiveram aumento na percepção dos conflitos nos relacionamentos interpessoais $(n=4)$; $5 \%$ indicaram o aumento no uso do tabaco $(n=3)$; e, por fim, recebemos respostas únicas ( $1,7 \%$ cada uma) que indicaram situações como $(n=1)$ : ausência de impactos, diminuição da ansiedade, pressão por redução salarial, saudades de ir ao local de trabalho, vontade de encontrar colegas e incertezas em relação ao futuro. Verifica-se, portanto, que a situação que se apresenta, com as mudanças e novas exigências, impactaram de diferentes maneiras os profissionais pesquisados, afetando seu bem-estar, qualidade de vida no trabalho e vida social.

Apresentamos a seguir as seis categorias, fruto do processo de análise de conteúdo realizado. As categorias foram elaboradas a posteriori, ou seja, resultaram da confrontação das considerações de três dos pesquisadores envolvidos na pesquisa e que realizaram individualmente as etapas de: repetição de leitura flutuante do corpus (conteúdo das questões abertas); identificação dos temas que mais se repetiam; codificação; enumeração; e, por último, a categorização (Bardin, 2011).

\section{Home Office ou Teletrabalho}

Verificou-se, no estudo realizado, que a maioria das organizações adotaram o home office como estratégia, visando à manutenção do distanciamento social e, ao mesmo tempo, continuar com as rotinas de trabalho e preservando empregos. O home office ou teletrabalho é definido por Filardi, Castro e Zanini (2020) como o trabalho que chega até o trabalhador e, por isso, difere-se da condição tradicional, em que o deslocamento é feito pelo trabalhador em direção ao local/posto de trabalho. Rocha e Armador (2018) definem teletrabalho como o trabalho feito à distância e o entendem como uma possibilidade de diversificação do modo de organizar a atividade profissional, permitindo que, por meios telemáticos, o trabalhador permaneça em sua residência, realizando de lá suas tarefas diárias.

Para a Sociedade Brasileira de Teletrabalho e Televendas (SOBRATT) (2016), há muitas vantagens resultantes da adoção do teletrabalho, tanto para as organizações quanto para os trabalhadores, posto que se pode verificar, por exemplo: economia de recursos naturais gerada pelo menor consumo nos locais de trabalho; diminuição de gastos com mobilidade 
e, consequentemente, redução do uso de combustíveis fósseis e da poluição no meio ambiente; melhoria da qualidade de vida dos trabalhadores, que passam a redirecionar melhor o tempo gasto com o trajeto casa-trabalho-casa e exercem suas atividades com maior flexibilidade e autonomia; redução do volume de carros no trânsito das cidades; entre outras.

Entretanto, se, por um lado, pode-se falar em vantagens resultantes do teletrabalho, pesquisas realizadas no Brasil e em diferentes países acenam também para a existência de desvantagens, em especial, sentidas pelos trabalhadores. O teletrabalho tem promovido uma invasão do trabalho no espaço de vida privada do trabalhador (Costa, 2007; 2013), que pode experimentar dificuldades em promover equilíbrio na relação trabalho-família, situação essa que foi relatada por $30 \%$ dos participantes do nosso estudo. Em home office, muitas vezes o volume de trabalho ultrapassa aquele da jornada normal do trabalho (Aderaldo, Aderaldo, \& Lima, 2017), ocupando espaços que deveriam ser destinados às questões pessoais e familiares. Os autores destacam também que, não raramente, os profissionais nessa modalidade lançam mão de recursos próprios para viabilizar a atividade profissional, ou seja, precisam arcar com os custos mensais de planos de conexão com internet, telefonia fixa e móvel e com gastos relativos a eventuais manutenções de equipamentos, fragilizando sua condição financeira.

O teletrabalho ainda tem sido apontado por pesquisadores como uma modalidade que expõe o trabalhador a um maior risco de informalidade e, consequentemente, de redução de garantias ou proteção a seus direitos (Aderaldo et al., 2017), além de ser um dos elementos responsáveis pelo fenômeno intitulado parcelarização do trabalho: um mesmo trabalhador pode, em função de dificuldades para lidar com o volume de trabalho e as exigências por desempenho, iniciar várias atividades ao mesmo tempo (parcelamento), sem, contudo, estabelecer fluxos que permitam adequada consecução das tarefas (Grisci \& Cardoso, 2014).

Neste período marcado pela pandemia de covid-19, queremos destacar o fato de que, para boa parte dos pesquisados, o teletrabalho assumiu um contorno ainda mais complexo. Nesse sentido, destacamos que a percepção de alguns participantes sobre o teletrabalho relaciona-se com o que Queiroga (2020) chama de teletrabalho compulsório, ou seja, que atravessou de modo abrupto e com uma urgência assaltante a relação já estabelecida entre o trabalhador-trabalho-organização, portanto, dotada de uma qualidade de vínculo e de inúmeras formas particulares de adaptação contextual. Assim sendo, o trabalho foi, literalmente, levado para casa, e de modo tão emergencial quanto temporário, pela grande maioria dos entrevistados, sem que tivessem tempo hábil para o planejamento e a execução de treinamentos visando sua capacitação. Essa situação trouxe desconforto emocional aos profissionais, que se viram inseridos num contexto de grandes transformações na dinâmica de seu trabalho e vida familiar. Martins, Aguiar e Bastos (2020, p. 49) apontam que "talvez, nunca antes, a fronteira entre o trabalho e a vida familiar foi tão tênue, praticamente inexistente".

\section{Distanciamento e Isolamento Social}

Os efeitos colaterais dessas medidas de isolamento e distanciamento social têm provocado alterações significativas na vida e rotina de pessoas, instituições/organizações de trabalho e da sociedade como um todo, fazendo com que se configurem verdadeiras crises no contexto da saúde pública e da economia (Estrada \& Koutronas, 2020). 
Os participantes do estudo relataram que, para além dos efeitos mobilizadores do distanciamento e isolamento social no âmbito do trabalho e de suas consequências para a economia de mercado, são também sentidos prejuízos em nível emocional-psicológico, resultantes, em sua grande maioria, da sensação de privação de liberdade, do confinamento ao espaço doméstico e da restrição de contato com pessoas que integram o círculo social existente, com os quais se mantém vínculos afetivos avaliados como significativos. Diante da pandemia e da ausência de métodos mais eficazes de tratamento e cura, e em função do isolamento e distanciamento social, que alteram o estado de "normalidade" da dinâmica de vida das pessoas, já se tem identificado, na população em geral, um aumento de sintomas relacionados à depressão, ansiedade e estresse, e, por isso, a necessidade de, também, providenciar formas de atenção à saúde mental das populações (Carvalho, Moreira, Oliveira, Landim, \& Rolim, 2020; Faro et al., 2020).

Incluem-se na lista dos elementos que desequilibram o estado de bem-estar psicológico da população em situação de distanciamento e isolamento social: sensação de confinamento/aprisionamento; tédio; confusão e insegurança diante das informações que circulam sobre a pandemia; frustração e raiva; incerteza quanto ao tempo de duração do confinamento, distanciamento social e pandemia; preocupações com escassez de alimentos; medo da perda de emprego e renda; preocupações relativas a um possível endividamento; medo de perder pessoas da família, em especial aqueles que se encontram em grupos de risco; sentimento de falta ou saudade das pessoas com as quais se tem vínculo afetivo significativo (Brooks et al., 2020; Dong \& Zheng, 2020; Weide, Vicentini, Araujo, Machado, \& Emuno, 2020; Faro et al., 2020; Schmidt, Crepaldi, Bolze, Neiva-Silva, \& Demenech, 2020).

\section{Alterações na Jornada de Trabalho}

Do público pesquisado, $85 \%(n=51)$ relataram mudanças em sua jornada de trabalho, seja por ocasião do aumento no volume de tarefas em função do teletrabalho compulsório, seja em função das decisões organizacionais para reduzir o risco de contágio entre os trabaIhadores, separando-os por turnos ou alternando escalas de dias de trabalho entre as equipes, para diminuir a necessidade de mobilidade de seus integrantes e, consequentemente, a permanência ou aglomeração de pessoas no espaço físico da organização. De forma interdependentemente relacionada ao tema das alterações nas jornadas de trabalho, demonstraram também, em suas respostas, preocupação com a já vivida situação de redução salarial em alguns setores do mercado, bem como receios relativos ao movimento a que se assiste em torno do aumento de demissões de trabalhadores e falências de empresas devido à crise econômica resultante da pandemia.

O Governo Federal, por meio de Medida Provisória (MP), instituiu o Programa Emergencial de Manutenção do Emprego e da Renda, que dispõe sobre medidas trabalhistas complementares para o enfrentamento do estado de calamidade pública e da emergência de saúde pública decorrente da pandemia de covid-19 (MP n. 936 [Brasil, 2020c]). A MP busca amenizar o momento enfrentado pelas organizações de trabalho e visa, sobretudo, a garantia dos empregos e a evitação de demissões em massa. Apesar de alguns impasses trabalhistas, a MP vem cumprindo seu papel de contribuir para amenizar os impactos negativos oriundos da pandemia, tanto para a economia como para a manutenção dos empregos. 
O contexto atual é de significativa retração na economia, com inevitáveis efeitos colaterais para as organizações de trabalho (podendo variar desde uso máximo de suas reservas ao financiamento de valores para custeio de compromissos com fornecedores e empregados junto aos bancos, até a falência) e para os trabalhadores (com volumoso aumento nos índices de desemprego e de atividades profissionais informais). Ao final de julho de 2020, o Instituto Brasileiro de Geografia e Estatística (IBGE, 2020) divulgou os dados da Pesquisa Nacional por Amostra de Domicílios Contínua Mensal (PNAD Contínua). A taxa de desemprego no Brasil subiu para 12,9\%, alcançando 12,7 milhões de pessoas. Em comparação ao trimestre anterior e, portanto, já sob os efeitos da pandemia, calcula-se um fechamento de 7,8 milhões de postos de trabalho. O cenário apresentado coloca os trabalhadores em uma situação de insegurança e desalento, que pode comprometer sua saúde física e mental (Marinho \& Camargo, 2017; Almeida, 2020).

\section{Alterações na Rotina de Trabalho e Vida}

O tema "alterações na rotina de trabalho e vida" se configurou como mais uma categoria de análise em nossa pesquisa. Seja por causa do teletrabalho compulsório, seja pelas alterações adotadas pelas organizações, assiste-se a uma verdadeira "desordem" nos fluxos tidos como normais/tradicionais de vida e trabalho de nossos participantes.

Deve-se considerar, como elemento agravante das alterações experimentadas em relação às rotinas de trabalho dos pesquisados, não somente fatos que os tocam diretamente e que se relacionam com sua condição de profissionais atuantes em diferentes segmentos e organizações. Assim sendo, consideramos também os fatos que, em decorrência de suas responsabilidades e inserções, sociais e em contextos familiares, apresentam-se como demandantes de sua atenção e, por isso mesmo, configuram-se como concorrentes em relação ao tempo antes dedicado quase que exclusivamente ao trabalho, ou pelo menos em período de tempo previamente planejado para ser dedicado ao trabalho (maternagem, paternagem, cuidados com idosos, trabalhos domésticos etc.) (Almeida, 2020; Queiroga, 2020).

Como indicado no estudo, o tempo para o trabalho e o tempo para outras vivências e atividades foram sobrepostos. O tempo para o lazer, suprimido, e o tempo para o autocuidado, secundarizado. O espaço da casa virou espaço de trabalho (quartos se transformaram em escritórios; mesas de refeição viraram mesas de trabalho e estudo). As demandas resultantes dos papéis de profissional concorrem com as demandas típicas da maternidade, paternidade e filiação (Martins et al, 2020). Com base em tal cenário, podemos postular que há, portanto, a oportunidade para a aproximação e ressignificação de vínculos afetivos intrafamiliares convivendo tenuamente com o risco de conflitos e intensificação de problemas relacionais. Pesquisadores como Vieira, Garcia e Maciel (2020) e Marques, Moraes, Hasselmann, Deslandes e Reichenheim (2020) apontam para o fato de que, por exemplo, o período equivalente às práticas de isolamento e distanciamento social tem sido também período de aumento nos registros de violência doméstica e familiar, com especial ênfase à violência contra a mulher.

Compreendemos que residem, sobre esta categoria de análise, elementos que interagem de forma interdependente e se modificam. Para alguns dos participantes da pesquisa, a situação não atinge de forma acentuada sua condição familiar e profissional, porém, para 
outros, e neste caso a maioria, pode trazer consequências prejudiciais à sua qualidade de vida e bem-estar, bem como de seus familiares.

\section{Condições Físicas e Materiais para o Trabalho: Dificuldades Diante do "Novo Normal"}

Por meio das respostas apresentadas obtivemos muitas sinalizações de dificuldades vivenciadas em relação às condições físicas e materiais para o trabalho no período e contexto pandêmico, em especial pelas exigências de adaptação ao uso dos equipamentos de proteção individual (EPI) e demais práticas de higiene pessoal, coletiva e ambiental, que passaram a ser necessárias. Muitas das medidas agora exigidas não faziam parte da rotina cotidiana do trabalho dos participantes e tiveram de ser incorporadas.

As normas relativas à saúde, higiene e segurança no trabalho ganharam maior visibilidade e, na visão dos participantes, isso tem gerado um certo desconforto, não obstante o entendimento de sua necessidade: a máscara facial dificulta a respiração, faz embaçar os óculos; o distanciamento dos colegas de trabalho gera uma sensação de impessoalidade desagradável; o rigor das medidas protetivas artificializa e esfria as relações; a redução dos atendimentos e encontros presenciais distancia as pessoas e torna o trabalho monótono; a redução das viagens de trabalho potencializa a sensação de rotina etc.

No entanto a proteção da saúde dos trabalhadores passa pela adequada utilização dos EPIs e exige de profissionais da área de GP uma atuação mais efetiva nas ações educativas, sensibilizando e treinando os trabalhadores em relação a seu uso, integrando um plano de resposta da organização à pandemia (Almeida, 2020; Secretaria de Inspeção do Trabalho, 2020).

\section{Impactos Financeiros Negativos: Demissões e Falências}

Uma parcela significativa dos respondentes $(35 \%, n=21)$ apontou para um fenômeno importante, considerando que, no referido cenário, as organizações para as quais trabalham estão enfrentando problemas financeiros e colocando em prática medidas para evitar a falência, sendo que a principal medida citada foi a redução do quadro de trabalhadores - demissões -, como é possível observar nos relatos dos participantes a seguir: "Se continuar nesse ritmo acredito que terá redução de salários e demissões"; "Muitas demissões por parte das empresas contratantes, além de fechamento de outras que não sobreviverão a quarente$n a^{\prime \prime}$, entre outros relatos que expressam conteúdo semelhante.

Sobre este tema, de acordo com os dados da Organização Internacional do Trabalho (2020), estima-se que, em nível mundial, o aumento do desemprego atinja entre 5,3 milhões e 24,7 milhões de pessoas a mais devido à pandemia da covid-19. Somando-se esses dados àqueles apresentados anteriormente em relação ao nível de desemprego que pode ocorrer no Brasil, o resultado será impactante, produzindo a ampliação das já gritantes diferenças sociais existentes (Porsse, Souza, Carvalho, \& Vale, 2020).

Outros autores (Ferreira Jr. \& Santa Rita, 2020; Gama Neto, 2020; Senhoras, 2020) entendem que os impactos econômicos serão bastante negativos em nível macro. Estes, por sua vez, impactam no nível micro, causando consequências financeiras às organizações, o que as obrigam a tomar medidas que evitem o seu fechamento, como as demissões citadas pelos participantes da presente investigação. Essa situação acentua a sensação de insegurança 
dos profissionais, podendo, em casos mais críticos, culminar em aumento da ansiedade e presença do estresse (Cullen, Gulati, \& Kelly, 2020; Schmidt et al., 2020).

\section{Considerações Finais}

O estudo apresentado procurou compreender como a pandemia da covid-19 tem influenciado as relações de trabalho, interpessoais e os trabalhadores, em especial aqueles que atuam nas áreas de GP das organizações. Participaram do estudo 60 diferentes profissionais atuantes nessa área (psicólogos, administradores, assistentes sociais, contadores, advogados etc.), o que demonstra a multidisciplinariedade que ela admite e que Ihe constitui enquanto identidade. As respostas foram analisadas, tanto pela ótica quantitativa como qualitativa, a partir de seis categorias de análise reveladas nas respostas dos participantes.

De forma geral, o estudo evidenciou a dificuldade que as organizações enfrentam na atualidade, necessitando a tomada de medidas para o melhor enfrentamento da situação. Nesse contexto, os profissionais de GP assumem protagonismo, considerando que as medidas de redução do contágio da covid-19 afetam diretamente todos os componentes da comunidade organizacional, ou seja, o público interno e externo, representado pelos trabalhadores, fornecedores, clientes e parceiros.

São esses profissionais que estarão na "linha de frente" das organizações, capacitando e orientando os trabalhadores para as necessárias medidas e os novos procedimentos de trabalho. No entanto esses profissionais também estão sujeitos aos impactos negativos do momento, como revelou o estudo: $85 \%(n=51)$ dos participantes relataram estar experimentando quadros de desgaste mental e exaustão emocional, com dificuldades de equilibrar as demandas do trabalho com outras importantes para o equilíbrio emocional, como as demandas familiares, por exemplo. O quadro apresentado coloca em alerta as organizações de trabalho, que deverão ser impactadas pela situação de saúde mental dos seus profissionais, com prováveis impactos em seu desempenho e em aumento nos índices de absenteísmos e rotatividade, mesmo quando o cenário de pandemia terminar. Esses fatores sinalizam para a necessidade de maiores estudos para elucidar os fenômenos que se apresentam e aqueles que estão por vir, em especial no âmbito da saúde mental dos trabalhadores.

Como esta pesquisa incluiu indistintamente profissionais atuantes na área de gestão de pessoas (psicólogos, administradores, assistentes sociais, contadores, economistas, advogados etc.), pode ser interessante, em estudos e pesquisas posteriores, separar o público-alvo por grupos de identidade profissional (área de formação), de modo que se possa comparar e analisar eventuais diferenças nos resultados (ressonâncias da pandemia no âmbito da saúde física e mental, devido às mudanças e adaptações necessárias ao enfrentamento desta) e a provável influência do aparato teórico-metodológico construído pelo participante via processo de formação acadêmica e experiência profissional, o que se identifica como limite no presente estudo.

O presente estudo não teve a pretensão de esgotar ou mesmo concluir sobre o assunto, considerando sua abrangência e complexidade, mas sim contribuir para a compreensão das consequências que esse momento nos traz, tanto para as relações de trabalho como para as relações interpessoais profissionais estabelecidas. Todavia detectou-se claramente, por meio desta pesquisa, que o contexto pandêmico agravou um cenário de relação homem-trabalho-organização em que já se presentificavam medos, incertezas, inseguranças 
e vulnerabilidades; experiências subjetivas das quais não estão isentos os profissionais atuantes em gestão de pessoas, inclusive os psicólogos organizacionais e do trabalho.

\section{Referências}

Aderaldo, I. L., Aderaldo, C. V. L., \& Lima, A. C. (2017). Aspectos críticos do teletrabalho em uma companhia multinacional. Cadernos EBAPE.BR, 15(esp.), 511-533. doi:http://dx.doi. org/10.1590/1679-395160287

Almeida, I. M. (2020). Proteção da saúde dos trabalhadores da saúde em tempos de covid-19 e respostas à pandemia. Revista Brasileira de Saúde Ocupacional, 45(e17). doi:https:// dx.doi.org/10.1590/scielopreprints.140

Azevedo, C. E. F., Oliveira, L. G. L., Gonzalez, R. K., \& Abdalla, M. M. (2013). A estratégia de triangulação: objetivos, possibilidades, limitações e proximidades com o pragmatismo. VI Encontro de Ensino e Pesquisa em Administração e Contabilidade, Brasília-DF. Recuperado de http://www.anpad.org.br/admin/pdf/EnEPQ5.pdf

Bardin, L. (2011). Análise de conteúdo. São Paulo: Edições 70.

Brasil. Ministério da Saúde. (2020a). Secretaria de Vigilância em Saúde. Sobre a doença. Retirado de https://coronavirus.saude.gov.br/sobre-a-doenca\#interna

Brasil. Ministério da Saúde. (2020b). Secretaria de Vigilância em Saúde. Painel coronavírus. Retirado de https://covid.saude.gov.br/

Brasil. (2020c). Medida Provisória 936 (10 de abril). Institui o Programa Emergencial de Manutenção do Emprego e da Renda. Brasília, DF. Recuperado de http://www. planalto. gov.br/ccivil_03/_ato2019-2022/2020/mpv/mpv936.htm

Brooks, S. K., Webster, R. K., Smith, L. E., Woodland, L., Wessely, S., Greenberg, N., \& Rubin, G. J. (2020). The psychological impact of quarantine and how to reduce it: Rapid review of the evidence. The Lancet, 395(10227), 912-920. doi:https://doi.org/10.1016/ S0140-6736(20)30460-8

Carvalho, P. M. M., Moreira, M. M., Oliveira, M. N. A., Landim, J. M. M., \& Rolim, M. L., Neto (2020). The psychiatric impact of the novel coronavirus outbreak. Psychiatry Research, 286(112902), 1-2. doi: http://dx.doi.org/10.1016/j.psychres.2020.112902

Castro, B. L. G., Oliveira, J. B. B., Morais, L. Q., \& Gai, M. J. P. (2020). Covid-19 e organizações: Estratégias de enfrentamento para redução de impactos. Revista Psicologia Organizações e Trabalho, 20(3), 1059-1063. https://dx.doi.org/10.17652/rpot/2020.3.20821

Costa, I. S. A. (2007). Teletrabalho: subjugação e construção de subjetividades. Revista de AdministraçãoPública,41(1),105-124.doi:https://doi.org/10.1590/S0034-76122007000100007

Costa, I. S. A. (2013). Controle em novas formas de trabalho: Teletrabalhadores e o discurso do empreendedorismo de si. Cadernos EBAPE.BR, 11(30), 462-474. Retirado de http:// bibliotecadigital.fgv.br/ojs/index.php/cadernosebape/article/viewFile/11655/10602

Cullen, W., Gulati, G., \& Kelly, B. D. (2020). Mental health in the covid-19 pandemic. QJM: An International Journal of Medicine, 113(5), 311-312. doi:https://doi.org/10.1093/qjmed/ hcaa110

Dong, M., \& Zheng, J. (2020). Letter to the editor: Headline stress disorder caused by Netnews during the outbreak of covid-19. Health Expect, 23(2), 259-260. doi:https://doi. org/10.1111/hex.13055 
Estrada, M. R., \& Koutronas, E. (2020). The Application of the 2019-nCoV Global Economic Impact Simulator (the 2019-nCoV-GEI-Simulator) in China. SSRN Eletronic Journal. doi:http://dx.doi.org/10.2139/ssrn.3542817

Faro, A., Bahiano, M. A., Nakano, T. C., Reis, C., Silva, B. F. P., \& Vitti, L. S. (2020). Covid-19 e saúde mental: A emergência do cuidado. Estudos de Psicologia (Campinas), 37(e200074). doi:https://doi.org/10.1590/1982-0275202037e200074

Ferreira Jr., R. R., \& Santa Rita, L. P. (2020). Impactos da covid-19 na economia: Limites, desafios e políticas. Cadernos de Prospecção, 13(2), 459-476. doi:http://dx.doi.org/10.9771/ cp.v13i2.COVID-19.36183

Filardi, F., Castro, R. M. P., \&Zanini, M. T. F. (2020). Vantagens e desvantagens do teletrabalho na administração pública: Análise das experiências do Serpro e da Receita Federal. Cadernos EBAPE.BR, 18(1), 28-46. doi: https://doi.org/10.1590/1679-395174605

Fontanella, B. J. B., Ricas, J., \& Turato, E. R. (2008). Amostragem por saturação em pesquisas qualitativas em saúde: Contribuições teóricas. Cadernos de Saúde Pública, 24(1), 17-27. doi:https://doi.org/10.1590/S0102-311X2008000100003

Gama Neto, R. B. (2020). Impactos da covid-19 sobre a economia mundial. Boletim da Conjuntura (BOCA), 2(5), 113-127. doi: http://doi.org/10.5281/zenodo.3786698

Grisci, C. L. I., \& Cardoso, J. (2014). Experimentação do tempo e estilo de vida em contexto de trabalho imaterial. Cadernos EBAPE.BR, 12(4), 851-865. doi:https://doi. org/10.1590/1679-395114752

Instituto Brasileiro de Geografia e Estatística. (2020). Pesquisa Nacional por Amostra de Domicilios Contínua Mensal (PNAD Contínua): Maio/2020. Retirado de https://www.ibge. gov.br/estatisticas/sociais/trabalho/9171-pesquisa-nacional-por-amostra-de-domicilioscontinua-mensal.html?=\&t=destaques

Manandhar, S., Nakami, P., \& Baniya, N. (2020). A Novel Coronavirus Emerging in World Key Questions for Developing Countries and Under Developed Countries. North American Academic Research, 3(2), 473-497. doi: https://doi.org/10.5281/zenodo.3690311

Marinho, P. R. R., \& Camargo, M. L. (2017). O atual cenário da relação trabalhador-organizaçãotrabalho e os riscos à saúde do trabalhador. Revista Laborativa, 6(2), 115-125. Retirado de https://ojs.unesp.br/index.php/rlaborativa/article/view/1837/pdf

Marques, E. S., Moraes, C. L., Hasselmann, M. H., Deslandes, S. F., \& Reichenheim, M. E. (2020). A violência contra mulheres, crianças e adolescentes em tempos de pandemia pela covid-19: Panorama, motivações e formas de enfrentamento. Cadernos de Saúde Pública. 36(4), e00074420. doi:https://doi.org/10.1590/0102-311X00074420

Martins, L. B., Aguiar, C. V. N., \& Bastos, A. V. B. (2020). Covid-19: Seus Impactos nas Relações Trabalho-Família. In Queiroga, F. (Org.), Orientações para o home office durante a pandemia da COVID-19 (pp. 49-58) (Coleção O trabalho e as medidas de contenção da covid-19: Contribuições da Psicologia Organizacional e do Trabalho, v. 1). Porto Alegre: Artmed.

Organização Internacional do Trabalho. (2020). Como a covid-19 afetará o mundo do trabalho? Retirado de https://www.ilo.org/brasilia/noticias/WCMS_740753/lang--pt/ index.htm

Organização Mundial da Saúde. (2020a). Coronavirus disease (covid-19) Pandemic. Retirado de https://www.who.int/emergencies/diseases/novel-coronavirus-2019 
Organização Mundial da Saúde. (2020b). Getting your workplace ready for covid-19. Retirado de https://www.who.int/docs/default-source/coronaviruse/getting-workplace-ready-forcovid-19.pdf?ua=1

Pinheiro, V. (2020). América Latina e o emprego nos tempos de pandemia. OIT Brasília. Retirado de https://www.ilo.org/brasilia/noticias/WCMS_740030/lang--pt/index.htm

Porsse, A. A., Souza, K. B., Carvalho, T. S., \& Vale, V. A. (2020). Nota Técnica NEDUR-UFPR 012020: Impactos Econômicos da covid-19 no Brasil. Curitiba: NEDUR. Retirado de http:// www.nedur.ufpr.br/portal/wp-content/uploads/2020/04/nota-tecnica-nedur-ufpr-012020-impactos-economicos-da-covid-19-no-brasil.pdf

Queiroga,F.(Org.).(2020).Orientaçõesparaohomeofficeduranteapandemia dacovid-19(Coleção Otrabalhoeasmedidas decontençãodacovid-19:ContribuiçõesdaPsicologia Organizacionale doTrabalho,vol.1).PortoAlegre:Artmed.Retiradodehttps://www.sbpot.org.br/publicacoes/ livros/volume-1-orientacoes-para-o-home-office-durante-a-pandemia-da-covid-19/

Rocha, C. T. M., \& Amador, F. S. (2018). O teletrabalho: Conceituação e questões para análise.CadernosEBAPE.BR,16(1),152-162. doi:https://doi.org/10.1590/1679-395154516

Schmidt, B., Crepaldi, M. A., Bolze, S. D. A., Neiva-Silva, L., \& Demenech, L. M. (2020). Saúde mental e intervenções psicológicas diante da pandemia do novo coronavírus (covid-19). Estudos de Psicologia (Campinas), 37, e200063. doi:https://doi. org/10.1590/1982-0275202037e200063

Secretaria de Inspeção do Trabalho. (2020). Orientações gerais aos trabalhadores e empregadores em razão da pandemia da covid-19. Escola Nacional da Inspeção do Trabalho. Retirado de https://enit.trabalho.gov.br/portal/index.php/covid-19-coronavirus

Senhoras, E. M. (2020). Novo coronavírus e seus impactos econômicos no mundo. Boletim da Conjuntura (BOCA), 1(2), 39-42. doi: http://doi.org/10.5281/zenodo.3761708

Sociedade Brasileira de Teletrabalho e Televendas [SOBRATT]. (2016). Aprovada resolução que regulamenta o teletrabalho no Poder Judiciário. http://www.sobratt.org.br/index. php/14062016-aprovada-resolucao-que-regulamenta-o-teletrabalho-no-poderjudiciario/

Vendramini, C. M. M. (2016). Estatística e delineamento de pesquisa. In M. N. Baptista, \& D. C. CAMPOS. Metodologias de pesquisa em ciências: Análises quantitativa e qualitativa (pp. 208-228). São Paulo: LTC.

Vieira, P. R., Garcia, L. P., \& Maciel, E. L. N. (2020). Isolamento social e o aumento da violência doméstica: O que isso nos revela? Revista Brasileira de Epidemiologia, 23, e200033. doi:https://doi.org/10.1590/1980-549720200033

Weide, J. N., Vicentini, E. C. C., Araujo, M. F., Machado, W. L., \& Enumo, S. R. F. (2020). Cartilha para enfrentamento do estresse em tempos de pandemia. Porto Alegre: PUCRS/ Campinas: PUC-Campinas. Retirado de https://www.puc-campinas.edu.br/wp-content/ uploads/2020/04/cartilha-enfrentamento-do-estresse.pdf.pdf

Zanelli, J. C., Bastos, A. V. B., \& Rodrigues, A. C. A. (2014). Campo profissional do psicólogo em organizações de trabalho. In Zanelli, J. C., Bastos, A. V. B., \& Borges-Andrade, J. E. (Orgs.), Psicologia, Organizações e Trabalho no Brasil (pp. 549-582). Porto Alegre: Artmed. 
Recebido em: 17/11/2020

Última revisão: 29/01/2021

Aceite final: 04/02/2021

\section{Sobre os autores:}

Isabella Fernanda Rodrigues Felipe: Universidade Estadual Paulista "Júlio de Mesquita Filho" (UNESP), Faculdade de Ciências, Departamento de Psicologia, campus de Bauru, SP. Graduanda em Psicologia. E-mail: isabellafelipe97.if@gmail.com, Orcid: http://orcid.org/0000-0003-1244-1092

Vinícius Ramalho Medeiros: Universidade Estadual Paulista "Júlio de Mesquita Filho" (UNESP), Faculdade de Ciências, Departamento de Psicologia, campus de Bauru, SP. Graduando em Psicologia. E-mail: ramalho.mvinicius@gmail.com, Orcid: http://orcid.org/0000-0003-4607-6011

Mário Lázaro Camargo: Universidade Estadual Paulista "Júlio de Mesquita Filho" (UNESP), Faculdade de Ciências, Departamento de Psicologia e Programa de Pós-Graduação em Psicologia do Desenvolvimento e Aprendizagem, campus de Bauru, SP. Doutor em Psicologia. E-mail: mario.camargo@unesp.br, Orcid: http://orcid.org/0000-0003-1802-2382

Edward Goulart Júnior: Universidade Estadual Paulista "Júlio de Mesquita Filho" (UNESP), Faculdade de Ciências, Departamento de Psicologia, campus de Bauru, SP. Doutor em Psicologia. E-mail: edward.goulart@unesp.br, Orcid: http://orcid.org/0000-0002-4923-433X 
